\title{
Formulation of low cost artificial diet for Rrearing diamondback moth (Plutella xylostella L.)
}

\author{
M.T.M.D.R. Perera ${ }^{1^{*}}$, N. Senanayake ${ }^{2}$ and A.G.P.K. Premachandra ${ }^{2}$
}

\begin{abstract}
Use of biological agents for insect pest control is important to prevent degradation of natural resources and for consumers to have insecticide residue-free vegetables. Launching of such programmes to use parasites and parasitoids as biocontrol agents for insect pest control need rearing crop pests for parasitization purposes. Mass rearing of the diamondback moth (DBM; Plutella xylostella L.) in the laboratory using artificial diet is necessary to have a continuous supply of parasitoids for field release. Experiments were conducted in the Plant Quarantine Unit at Gannoruwa in Peradeniya, Sri Lanka using second instar DBM larvae. Nine artificial diets (AD1 to AD9) were formulated for rearing DBM and compared with cabbage (Brassica oleracea L.) leaves as the control. The AD2 to AD9 were formulated by modifying the expensive Biever and Boldt diet (AD1) with low cost material i.e. partial replacement of agar with gelatin, Casein with soya flour, Biever's yeast with ordinary yeast, raw wheat germ with 'semolina', Alphacel with filter papers, aureomycin with tetracycline, and linseed oil with vegetable oil. The results indicated that all the treatments had less survival rate of the DBM than the control. The percentage survival of the DBM on $A D 7, A D 4, A D 8$ and $A D 5$ diets were 48, 60, 60 and 72, respectively, compared to the control. The length of larvae was significantly higher $(p<0.05)$ in all treatments compared to control and $\mathrm{D}_{5}$ diets. The larval period in $\mathrm{AD}_{5}$ diet (8-12 days), though extended, was comparable to control (8-10 days). However, the mean weight of pupae reared on $\mathrm{AD}_{5}$ diet was significantly higher ( $p<0.05 ; 5.2 \mathrm{mg}$ ) than those of other diets. The number of males emerging from AD2, $A D 4, A D 8$ and $A D 9$ diets were higher than others whereas control and AD5 gave similar results ( $p>0.05$ ). Eggs laid per female were low in DBM reared in all artificial diets. The length of life cycle was low in AD5 (15-19 days) comparable to other diets. Overall results indicated that the $A D 4, A D 5$ and $A D 8$ were better than other diets while $A D_{5}$ was comparable to the control suggesting that this diet can be used for mass rearing of DBM in the laboratory.
\end{abstract}

Keywords: Artificial diet, Biever and Boldt diet, mass rearing, Plutella xylostella

\section{Introduction}

Cruciferous vegetables are economically important throughout the world. In Asia, they are grown in highlands often involving input-intensive practices. In recent years in Sri Lanka, the crucifer production was seriously hampered by a steady increase in insect pest populations, especially diamondback moth (DBM; Plutella xylostella L.), which feeds on the foliage and reduces the product quality. The DBM is one of the most destructive insect pests in the world reported in over 100 countries. Its short

\footnotetext{
1 Plant Quarantine Unit, Gannoruwa, Peradeniya, Sri Lanka

2 Department of Plant Science, Faculty of Agriculture, Rajarata University of Sri Lanka, Puliyankulama, Sri Lanka

* Corresponding author
} 
generation period, higher number of generations per year and each female laying up to 300 eggs are the reasons for its destructiveness. Generally, large quantities of insecticides have been used to control the DBM, resulting in development of resistance in the pest to almost all synthetic insecticides including Bacillus thuringiensis Berliner (Bt) formulations (Liu et al., 1995). The misuse and overuse of insecticides, enhancing the resistant development in vegetable growing areas of Sri Lanka has been reported by many scientists from the Department of Agriculture.

Talekar (1992) indicated that the estimated annual cost for managing DBM worldwide was about one billion US dollars. Though new insecticides are continuously developed to replace the existing ones, DBM has been able to develop resistance quickly to many of these insecticides (Nisin et al., 2000). Talekar and Shelton (1993) indicated that the use of broad spectrum insecticides also destroy natural enemies causing ecological imbalance and health hazards. Biological control, is one aspect of Integrated Pest Management (IPM) programmes, and has already proven to be an important pest management strategy for DBM control.

There are many egg, larval and pupal parasites and parasitoids found in nature to be used as biological control agents. Cotesia plutellae Kurdjumov (Hymonoptera: Braconidae) is an effective larval parasitoid of DBM, and deposits eggs in first three larval instars of DBM (Velasco, 1982). Mass rearing of DBM larvae is essential in biological insect pest control programmes. The objective of mass rearing is to produce large number of insects in the shortest possible time with minimum labor and space for which artificial feeding is also essential. The artificial diets for mass rearing of DBM larvae have been developed in many countries (Biever and Boldt, 1971; Hsiao and Hou, 1978), however, they are costly to prepare and use in Sri Lanka. Further those artificial diets have not been adaptable for the modern strains of DBM in some regions of the world (Dunhawoor and Abeeluck, 2003).

The ingredients used in the artificial diet preparations should be inexpensive and nutritionally optimal for growth and development of insects. The aim of this study was to evaluate the feasibility of using artificial diets formulated with some locally available low cost ingredients to rear DBM in the laboratory to use them to raise DBM cultures for mass rear its parasitoid, C. plutellae.

\section{MATERIALS AND METHODS}

\section{Location}

The study was conducted at the laboratory of Plant Quarantine Unit at Gannoruwa, Peradeniya, Sri Lanka ( $7^{\circ} 15^{\prime}$ latitude and $80^{\circ} 45^{\prime}$ longitude; altitude $482 \mathrm{~m}$ amsl; ambient temperature $28^{\circ} \mathrm{C}, \mathrm{RH} 80 \%$ at $08.00 \mathrm{hrs}$ ) during 2013.

\section{Establishment of DBM larval cultures}

The DBM larvae and pupae were collected from isolated cabbage (Brassica oleracea L.) fields at Nuwara Eliya, Thalathuoya, and the field of In-service Training Institute (ISTI), Gannoruwa, Sri Lanka. The larvae and pupae were reared on fresh cabbage 
leaves in separate boxes $(20 \mathrm{~cm} \times 15 \mathrm{~cm} \times 10 \mathrm{~cm})$ in the laboratory. Emerging adults were then placed in cages $(45 \mathrm{~cm} \times 45 \mathrm{~cm} \times 90 \mathrm{~cm})$ containing potted cabbage plants. The moths were fed with $10 \%$ bee honey solution soaked in absorbent cotton wool. Mated females were separated and allowed to lay eggs on fresh cabbage leaves in plastic boxes.

\section{Preparation of artificial diets}

The artificial diets were formulated based on Biever and Boldt diet. Agar, filter papers, normal papers, ascorbic acid, tetracycline, yeast, fresh and dry cabbage leaves, casein, cholesterol, gelatin, soya flour, sucrose, semolina, $\mathrm{KOH}$, coconut oil, mustard oil, propionic acid, citric acid and water in different combinations were used as ingredients. Eight artificial diets were formulated by modifying the Biever and Boldt diet using low cost ingredients (Table 1).

Table 1. Ingredient levels for one $\mathrm{kg}$ of diet formulation

\begin{tabular}{|c|c|c|c|c|c|c|c|c|c|c|}
\hline Ingredient & Unit & AD1* & $\mathrm{AD2}$ & $A D_{3}$ & AD4 & AD5 & AD6 & AD7 & AD8 & AD9 \\
\hline Agar & g & 23.0 & 23 & 8 & 6 & 6 & 2 & - & 5 & 4 \\
\hline Filter papers & g & - & 5 & 5 & 5 & 5 & 4 & - & 5 & - \\
\hline Alphacel & $g$ & 5.0 & - & - & - & - & - & - & - & . \\
\hline Ascorbic acid & $\mathrm{ml}$ & 4.0 & 4. & 4 & 4 & 4 & 4 & - & 4 & 4 \\
\hline Aureomycin & g & 1.5 & - & - & - & - & - & - & - & - \\
\hline Tetracycline & $g$ & - & 1 & 2 & 2 & 2 & - & 2 & 2 & 2 \\
\hline Yeast & $G$ & - & 16 & 5 & 34 & 34 & 35 & 34 & 35 & 36 \\
\hline Cabbage (dry) & $G$ & 12.5 & 67 & 2 & - & - & - & - & - & - \\
\hline Cabbage (fresh) & $G$ & - & - & 60 & 340 & 600 & 340 & 336 & 526 & 528 \\
\hline Casein & G & 35.0 & 35 & 15 & 30 & 35 & 15 & 10 & 4 & 12 \\
\hline Choline chloride & g & 10.0 & - & - & - & - & - & - & - & - \\
\hline Cholesterol & $g$ & - & 3 & 2 & 4 & 3 & 4 & 4 & 14 & 12 \\
\hline Gelatin & g & - & - & 14 & 20 & 17 & 24 & 30 & 19 & 18 \\
\hline Soya flour & g & - & - & 35 & 20 & 30 & 64 & 70 & 40 & 50 \\
\hline Sucrose & g & 35.0 & 30 & 5 & 30 & 35 & 30 & 30 & 28 & 28 \\
\hline Wessons salt & $g$ & 10.0 & 10 & 5 & 4 & 4 & 4 & 4 & 4 & 4 \\
\hline Wheat germ & g & 30.0 & - & - & - & - & - & - & - & - \\
\hline Semolina & g & - & - & 20 & 44 & 100 & 40 & 48 & 60 & - \\
\hline Methyl P & $g$ & 1.5 & - & - & - & - & - & - & - & - \\
\hline $\mathrm{KOH}$ & $\mathrm{ml}$ & 5.0 & 5 & 3 & 4 & 5 & 5 & 12 & 5 & 5 \\
\hline Linseed oil & $\mathrm{ml}$ & 12.0 & & & & & & & & \\
\hline Coconut oil & $\mathrm{ml}$ & - & - & 7 & 3 & 7 & 12 & 14 & 14 & 14 \\
\hline Surfactant & $\mathrm{ml}$ & - & 10 & 10 & 4 & 4 & 4 & 4 & 4 & 4 \\
\hline Formaldehyde & $\mathrm{ml}$ & 0.5 & - & - & - & - & - & - & - & - \\
\hline Vitamin B & g & 1.0 & - & - & - & - & - & - & - & . \\
\hline Water & $\mathrm{ml}$ & 814.0 & 791 & 798 & 446 & 125 & 413 & 402 & 231 & 279 \\
\hline
\end{tabular}


The ingredients were weighed separately. Dry ingredients were mixed with hot water $\left(70{ }^{\circ} \mathrm{C}\right)$ in a blender. Agar was dissolved in a beaker at $80{ }^{\circ} \mathrm{C}$, cooled to $70{ }^{\circ} \mathrm{C}$ and added to the mixture in a blender. Filter papers, cabbage leaf powder and the heated wheat germ were mixed thoroughly. Thereafter, cholesterol dissolved in ether was added to the mixture, stirred well to evaporate ether. Sucrose, casein and Wesson's salt mixture were added to the melted agar solution, and the resulting solution was poured together with the solid mixture into a blender. Then, aureomycin, ascorbic acid and vitamin B were added immediately. The whole mixture was ground thoroughly in the blender for 1-2 $\min$ enabling it to be dispensed. The leaf powder is usually prepared from fresh cabbage leaves by drying at $60{ }^{\circ} \mathrm{C}$ for $24 \mathrm{~h}$ and then grinding in a blender, and the powder is then screened through a 100-mesh sieve before storing at low temperature. The prepared diets were dispensed into autoclaved Petri dishes.

The diets were cut into pieces of one cubic centimeter and one cube of each diet was placed in a sterilized Petri dish individually, where ten $2^{\text {nd }}$ instars (three-four day old) DBM larvae were placed. Fresh cabbage leaves were used as the control treatment. The diet cubes and cabbage leaves in the control were replaced at every two day intervals. Complete randomized design with five replicates was used in this experiment. Daily counts of the number of surviving larvae, days to attain pupal stage, weight of pupae, number of emerged moths, number of males and females and number of eggs laid by the females were taken.

Data were analyzed by the Analysis of Variance (ANOVA) using SAS software package. Duncan's New Multiple Range Test $(p=0.05)$ was used for mean separation.

\section{Results and Discussion}

\section{Percentage survival and mean lifespan of $P$. xylostella}

The percentage survival of larvae of DBM was significantly higher $(p<0.05)$ in the control, AD5, AD4, and AD8 treatments than the others (Table 2). However, the treatment AD1 (Biever and Boldt diet), gave a significantly lower $(p<0.05)$ survival. Dunhawoor and Abeeluck (2003) have also reported that some feeds are not adaptable for the modern strains of DBM in some regions of world. Larvae on the control treatment had the highest survival percentage (Table 2). The larval mortality in different treatments may be attributed to various factors such as injury to larvae while transferring to Petri dishes, during replacement of diet cubes and also due to differences in nutrient composition of the diet formulations.

There were no significant differences ( $p>0.05)$ among treatments on the mean lifespan (Table 2). The highest mean lifespan of larvae to adult was observed in AD1 treatment. However, the larvae fed on cabbage leaves (control) had the shortest mean lifespan compared to other treatments except AD5, which indicates that the other diet formulations are deficient nutritionally to support the normal growth and development of the larvae. 
Table 2. Percentage survival and mean lifespan of P. xylostella under different treatments

\begin{tabular}{lcc}
\hline Treatment & Percentage survival & Mean lifespan \\
\hline AD1* (Biever and & $12^{\mathrm{e}}$ & $24^{\mathrm{a}}$ \\
Boldt) & $28^{\mathrm{d}}$ & $22^{\mathrm{a}}$ \\
AD2 & $32^{\mathrm{d}}$ & $23^{\mathrm{a}}$ \\
AD3 & $60^{\mathrm{b}}$ & $16^{\mathrm{a}}$ \\
AD4 & $70^{\mathrm{ab}}$ & $15^{\mathrm{a}}$ \\
AD5 & $40^{\mathrm{cd}}$ & $16^{\mathrm{a}}$ \\
AD6 & $48^{\mathrm{c}}$ & $17^{\mathrm{a}}$ \\
AD7 & $60^{\mathrm{b}}$ & $17^{\mathrm{a}}$ \\
AD8 & $32^{\mathrm{d}}$ & $17^{\mathrm{a}}$ \\
AD9 & $90^{\mathrm{a}}$ & $15^{\mathrm{a}}$ \\
Control & & \\
CV\% & 17.1 & 11.3 \\
\hline
\end{tabular}

*AD = artificial diet; within each column, means followed by the same letter are not significantly different by the DMRT at $\mathrm{p}=0.05$.

\section{Number of days to attain pupal stage and mean weight of pupae of $P$. xylostella}

Significantly lower $(p<0.05)$ larval periods were observed in the control and the artificial diets AD5, AD4 and AD8 (Table 3). The lowest larval period was reported in the control and the highest in AD1 (Biever and Boldt diet). Significantly higher $(p<0.05)$ larval periods were also observed when the pest was fed with AD2 and AD3 treatments. There were no significant difference ( $p>0.05)$ among Biever and Boldt (AD1), AD2, AD3 and AD9 treatments. The larval period was extended in Biever and Boldt diet indicating its unsuitability under local environmental conditions and/or the physiological status of the DBM strains available in Sri Lanka.

The mean weight of pupae was significantly higher $(p<0.05)$ in the control and was similar to that of $A D_{2}, A D 3, A D 4$ and $A D_{5}$ treatments. There were no significant differences ( $p>0.05$ ) among the AD6, AD7, AD8 and AD9 treatments and AD1, AD4, $A D 5$ and AD8 treatments (Table 3). The highest mean pupal weight was obtained in the control indicating that fresh cabbage leaves are the ideal food for DBM caterpillars for its normal growth and development.

The Biever and Boldt diet gave the longest pupal period and intermediate weight of the pupa indicating its unsuitability under local conditions. Treatment AD9 gave the lowest pupal weight though its pupal period was comparable to AD5 treatment, which was similar to the control in all other parameters. The difference between these two formulations was that ADg formulation did not have raw wheat germ and Alphacel or its low cost alternatives, semolina and filter papers, indicating the probable importance of these ingredients in normal growth and development of the larvae. It is further interesting to note that these two treatments did have almost equal amount of fresh cabbage leaves in the formulations. 
Table 3. Mean number of days to attain pupal stage and mean weight of pupae of $P$. xylostella $(\mathrm{n}=10)$ on different treatments

\begin{tabular}{lcc}
\hline Treatment & Days to attain pupal stage & Weight of pupae $(\mathrm{mg})$ \\
\hline AD1(Biever and Boldt) & $11.7^{\mathrm{a}}$ & $4.40^{\mathrm{b}}$ \\
AD2 & $11.6^{\mathrm{a}}$ & $4.48^{\mathrm{a}}$ \\
AD3 & $11.3^{\mathrm{a}}$ & $4.61^{\mathrm{a}}$ \\
AD4 & $8.7^{\mathrm{c}}$ & $4.45^{\mathrm{abc}}$ \\
AD5 & $8.2^{\mathrm{c}}$ & $5.00^{\mathrm{ab}}$ \\
AD6 & $9.4^{\mathrm{b}}$ & $4.05^{\mathrm{dc}}$ \\
AD7 & $10.0^{\mathrm{b}}$ & $4.10^{\mathrm{dc}}$ \\
AD8 & $8.4^{\mathrm{c}}$ & $4.18^{\mathrm{bcd}}$ \\
AD9 & $10.3^{\mathrm{ab}}$ & $3.35^{\mathrm{d}}$ \\
Control & $8.0^{\mathrm{c}}$ & $5.20^{\mathrm{a}}$ \\
& & \\
CV\% & 7.4 & 6.4 \\
\hline
\end{tabular}

${ }^{*} \mathrm{AD}=$ artificial diet; within each column, means followed by the same letter are not significantly different by the DMRT at $\mathrm{p}=0.05$.

\section{Sex ratio and fecundity of $P$. xylostella}

Generally in insects, male to female sex ratio is closer to 1:1 (Hamilton, 1967). The results of the present study indicated that male to female ratio of the control, $A D 3$, AD5, AD6, and AD7 were 1:1 (Table 4). However, a higher number of males was recorded in artificial diets $A D 2$ and $A D 9$. The difference in these two treatments compared to others is the absence of wheat germ or semolina indicating importance of these ingredients in artificial diets. The Biever and Boldt diet (AD1), AD4 and AD8 gave intermediate values for the number of emerged males. This shows that the composition of nutrients in standard Biever and Boldt diet favours production of more males, which is a disadvantage for mass rearing purposes. Varied sex ratios among treatments could be attributed to the differences in nutrient composition (Hamilton, 1967).

The highest average number of eggs laid per female was observed in the control treatment and the lowest was in the Biever and Boldt treatment (AD1). The average number of eggs laid per female in $\mathrm{AD}_{5}$ treatment was comparable to the control (Table 4). Larvae fed with fresh cabbage leaves; AD4, AD5, and AD8 diets had higher fecundity, which indicates that the nutrient composition is comparable to each other and the control. It is also interesting to note that egg laying capacity of DBM fed with AD1 (Biever and Boldt diet) was lower, which may be attributed to the differences in environment conditions and/or physiological status of DBM under local conditions.

The main objectives of mass rearing are to produce the maximum number of physically and biologically sound insects at the shortest possible time. Therefore, the insects should be adapted to artificial feeding for mass rearing purposes. In this experiment, the Beiver and Boldt diet (AD1) was taken as the standard artificial diet and others were formulated based on it. However, the standard Beiver and Boldt diet performed poorly in contrary to the expectations and was significantly lower in 
all parameters compared to the control treatment, where the fresh cabbage leaves were used. Further, the Beiver and Boldt diet resulted in low fecundity indicating its unsuitability as an artificial diet to rear DBM in Sri Lanka for mass rearing of C. plutellae. This could be attributed to the differences in environment conditions, where the experiments were conducted and/or to the differences in the physiological status of insects locally available and used in the experiments. Further, the nutrient composition of the artificial diets should be optimal for the normal growth and development of the insects if they were to be used for mass rearing purposes. Friend (1958) also surmised that the artificial diets should satisfy the chemical, physical and nutritional requirement of the insects for optimal growth and development.

Table 4. Mean values of male:female ratio and the fecundity of P. xylostella

\begin{tabular}{lcc}
\hline Treatments & Male:Female Ratio & $\begin{array}{c}\text { Average No. of } \\
\text { eggs/Female }\end{array}$ \\
\hline AD1 (Biever and Boldt) & $2: 1$ & $48^{\mathrm{d}}$ \\
AD2 & $4: 3$ & $52^{\mathrm{d}}$ \\
AD3 & $1: 1$ & $76^{\mathrm{bc}}$ \\
AD4 & $2: 1$ & $115^{\mathrm{b}}$ \\
AD5 & $1: 1$ & $171^{\mathrm{a}}$ \\
AD6 & $1: 1$ & $54^{\mathrm{d}}$ \\
AD7 & $1: 1$ & $63^{\mathrm{c}}$ \\
AD8 & $2: 1$ & $124^{\mathrm{b}}$ \\
AD9 & $3: 1$ & $65^{\mathrm{c}}$ \\
Control & $1: 1$ & $180^{\mathrm{a}}$ \\
CV\% & & 18.6 \\
\hline
\end{tabular}

*AD = artificial diet; within each column, means followed by the same letter are not significantly different by the DMRT at $\mathrm{p}=0.05$.

Among the parameters measured, sex ratio is an important factor in mass rearing process of any insect in order to have large number of female insects at a given time. Sex ratio among treatments was different probably due to feeding insects with artificial diets having different nutrient compositions. Diets AD3, AD5, AD6 and AD7 and control gave normal sex ratio of 1:1. High fecundity is another important factor for mass rearing insects in the laboratory as it is possible to produce large number of insects within a short period. Fecundity among treatments having sex ratio of 1:1, $A D 5$ treatment was the only treatment which had no significant difference ( $p>0.05)$ to the control treatment.

The number of days to attain pupal stage varied from 8 to 12 days in the laboratory, where the control treatment took eight days. When the number of days to attain the pupal stage is lower, the number of generations that could be obtained per year will be higher. Similar results were obtained when DBM was fed on artificial diets in Taiwan at ambient temperatures of $23+1{ }^{\circ} \mathrm{C}$ and $60+5 \% \mathrm{RH}$ conditions $(\mathrm{Wu}$, 
1968; Hsiao and Hou, 1978). Further, control and AD5 treatments took the same minimum number of days to attain pupal stage indicating the nutrition composition of the cabbage leaves and AD5 diet are nutritionally similar if not equal.

Of the nine artificial diets, the $\mathrm{AD}_{5}$ treatment gave results closer to the control treatment and was not significantly different $(p>0.05)$ in all parameters. Therefore it can be concluded that AD5 diet can be used for rearing DBM. However, the survival rate of $\mathrm{DBM}$ larvae in $\mathrm{AD}_{5}$ treatment was lower (70\%) than the control treatment (90\%) though not statistically significant ( $p>0.05)$. As other parameters of $A D 5$ showed similar results to that of the control, the lower survival could probably be attributed to the disturbance caused to the larvae while handling.

Results also indicated that some of the low cost ingredients used in formulation of artificial diets namely, filter paper, gelatin, Tetracycline, semolina, coconut oil, normal yeast, and soya flour did not have any harmful effect on parameters measured on growth and development of DBM. However, the best results shown by AD5 treatment had its composition different to Biever and Boldt diet (AD1) as some of the ingredients were replaced successfully by low cost material. These replacements are a partial replacement of agar with gelatin, raw wheat germ with semolina, Alphacel with filter papers, aureomycin with tetracycline and linseed oil with coconut oil.

\section{Conclusion}

The artificial diet $\mathrm{AD}_{5}$ is better than all other diet formulations and comparable to the control. Biever and Boldt diet performed poorly under the local environmental conditions and the physiological status of locally available DBM. The expensive ingredients can be partially or completely be replaced by locally available low cost ingredients, e.g. agar can be partially replaced with gelatin, the total requirement of Alphacel with filter paper, aureomycine with tetracycline, raw wheat germ with semolina, and linseed oil with coconut oil.

\section{Acknowledgement}

The authors wish to thank the Sri Lanka Council for Agricultural Research Policy (SLCARP) for their financial support to carry out this study, the Department of Agriculture (DOA), Sri Lanka for facilitating the research to be done at the laboratory of the Plant Quarantine Unit (PQU).

\section{References}

Biever, K.D. and Boldt, P.E. (1971): Continuous Laboratory rearing of the diamondback moth and related biological data. Ann. Entomol. Soc. Amer. 64: 651-655. 
Dunhawoor, C. and Abeeluck, D. (2003): Rearing of the Diamondback Moth Plutella xylostella L. (Lepidoptera: plutellidae) on artificial diet in the laboratory In Annual Meeting of Agricultural Scientists (AMAS). pp 133-139 Reduit, Mauritius.

Friend, W.G. (1958): Nutritional requirement of phytophagus insects. Ann. Rev. Entomol. 3: 57-74.

Hamilton, W D. (1967): Extra ordinary sex ratios. Science 156: 477-488.

Hsiao, J.H. and Hou, R.F. (1978): Artificial rearing of diamondback Moth, Plutella xylostella. L on a semi-synthetic diet. Entomol. J. 17: 97-102.

Liu, Y.B., Tabashnik, B.E. and Johnson, M.W. (1995): Larval age effects resistance to Bacillus thuringiensis in diamondback moth (Lepidoptera: Plutellidae). J. Econ. Entomol. 88: 788792.

Nisin, K.D., Mo J. and Miyata, T. (2000): Decrease susceptibilities of four field populations of diamondback moth Plutella xylostella L. (Lepidoptera: Yponomeutidae). Appl. Entomol. Zool. 35: 591- 595 .

Talekar, N.S. and Shelton A.M. (1993): Biology, ecology and management of diamondback moth. Ann. Rev. Entomol. 38: 64-70.

Talekar, N.S. (1992): Proceedings of the international workshop on diamondback moth and other crucifer pests. pp 78-84, AVRDC, Taiwan.

Velasco, L.R.I. (1982): Life history of Apenteles plutellae Kurdj. (Brachonidae) a parasitoid of the diamondback moth. Phillip. Entomol. 5:385-399.

Wu, K.C. (1968): Bionomic study of Plutella maculipennis Curtis. (In Chinese). Agric. Res. 7: 1721. 
Perera et al. 